\title{
Low-Grade Systemic Inflammation Profile, Unrelated to Homocysteinemia, in Obese Children
}

\author{
Emanuel V. Economou, ${ }^{1}$ Ariadne V. Malamitsi-Puchner, ${ }^{2}$ Christos P. Pitsavos, ${ }^{3}$ \\ Evangelia E. Kouskouni, ${ }^{1}$ Ioanna Magaziotou-Elefsinioti, ${ }^{4}$ and George Creatsas ${ }^{5}$ \\ ${ }^{1}$ Hormone Laboratory, 2nd Clinic for Obstetrics and Gynecology, Aretaieion Hospital, University of Athens, 11528 Athens, Greece \\ ${ }^{2}$ Division of Neonatology, 2nd Clinic for Obstetrics and Gynecology, Aretaieion Hospital, University of Athens, 11528 Athens, Greece \\ ${ }^{3}$ Department of Cardiology, Hippokration Hospital, University of Athens, 11527 Athens, Greece \\ ${ }^{4}$ Department of Pediatrics, Tzaneion Hospital, 18536 Pireaus, Greece \\ ${ }^{5} 2$ nd Clinic for Obstetrics and Gynecology, Aretaieion Hospital, University of Athens, 11528 Athens, Greece
}

Received 1 August 2005; accepted 5 September 2005

To investigate in prepubertal obese children (POC) the profile of chronic low-grade systemic inflammation (CLGSI) and its relation to homocysteinemia, 72 POC were evaluated for serum C-reactive protein (CRP) and amyloid A (SAA) levels, both markers of CLGSI, and plasma levels of total homocysteine (tHcy), an independent risk factor for adult atherosclerosis, in comparison to 42 prepubertal lean children (PLC). The main observations in POC were higher CRP levels compared to PLC, positive association of SAA levels to CRP levels, no association of CRP or SAA levels to tHcy levels. Thus, in POC, positively interrelated to each other, elevated CRP and unaltered SAA levels reveal a unique profile of the CLGSI, not explaining homocysteinemia-induced risk for future atherosclerosis.

\section{INTRODUCTION}

Several cardiovascular risk factors are present in obese children, among which are chronic low-grade systemic inflammation and homocysteinemia [1].

Obesity is now widely accepted as a promoter of a chronic low-grade inflammatory reaction favoring the development of atherosclerosis and cardiovascular disease [2], in both children and adolescents $[3,4]$ who are free of other pathological conditions. Chronic lowgrade systemic inflammation, usually a persistent but more sublte than acute phase inflammatory response, can be measured by circulating C-reactive protein (CRP) and serum amyloid A (SAA) which may also have direct proinflammatory actions $[5,6]$. They both are nonspecific acute phase reactants, primarly synthesized in liver and at least equally sensitive to reflect chronic low-grade systemic vascular inflammation [7]. Moreover, highly sensitive newer assays for CRP and SAA can now detect previously unnoticed chronic low-grade systemic inflammation $[8,9]$. In obese subjects, hepatic biosynthesis and maintenance of circulating CRP and SAA is up-regulated

Correspondence and reprint requests to Emanuel V. Economou, Hormone Laboratory, 2nd Clinic for Obstetrics and Gynecology, Aretaieion Hospital, University of Athens, 11528 Athens, Greece; eveconom@otenet.gr by adipocytokines, such as interleukins 1 and 6 , tumor necrosis factor $\mathrm{A}$, and, for some authors, leptin, all secreted by adipose tissue [10]. However, although equally sensitive and similarly up-regulated, CRP and SAA are differentially determined in circulation. Circulating CRP levels are determined by both environmental factors and a moderate but significant degree of heritability while circulating SAA levels are determined exclusively by environmental factors [11, 12]. Moreover, several studies describe differential, population-specific, local or systemic response of CRP and SAA to chronic low-grade vascular inflammation $[5,13,14,15]$. These variations could indicate the existence of positive and negative control mechanisms that permit, independently, the induction of CRP or SAA levels to fulfill the host-specific low-grade inflammatory response and it is likely to be of relevance to the causation of future disease.

Mild to moderate elevation of circulating total homocysteine (tHcy), commonly referred to as homocysteinemia, is generally considered to be an independent risk factor for atherosclerosis [16]. The strength of the association between an elevated tHcy and cardiovascular disease seems to vary among populations [17]. In adults, considerable evidence has been accumulated to support that the link between tHcy and atherothrombosis cannot be explained by associations of tHcy with the chronic low-grade inflammation estimated from serum CRP [13]. Confirming previous observations by others $[18,19]$, 
we recently demonstrated that, independently of age and sex, a modest elevation of plasma tHcy is, at least, indirectly associated with childhood obesity $[8,20]$. However the molecular pathogenesis underlying all these observations in vivo has yet to be fully defined.

Prepubertal obese children (POC) represent a unique study population in that limitation of confounding by inflammation-related diseases and subclinical cardiovascular disease is expected. To our knowledge no data exist in prepubertal childhood obesity exploring the profile of chronic low-grade inflammation with regard not only to circulating CRP but also to circulating SAA and moreover, investigating a possible relationship among tHcy and circulating markers of this specific type of chronic lowgrade systemic inflammation. The present study intended to investigate in POC the profile of chronic low-grade systemic inflammation as estimated by acute phase reactants CRP and SAA, their possible interrelation, and moreover, a possible association among CRP, SAA, and tHcy. This would help to determine whether there is some underlying process related to this specific type of inflammation that might be relevant to homocysteine-mediated future atherosclerosis in POC. The findings of this study suggest that a differential profile of chronic low-grade systemic inflammation, in that, although interrelated to each other, only CRP levels which are negatively related to high-density lipoprotein (HDL) cholesterol, not SAA levels which are unrelated to HDL cholesterol, are elevated, is observed in prepubertal childhood obesity and this is unlikely to be the link between prepubertal childhood obesity-associated homocysteinemia and adulthood atherosclerosis.

\section{MATERIALS AND METHODS}

\section{Participants}

The study, approved by the Ethics Committee of our General Hospital, involved 72 consecutive prepubertal obese children (POC) and lasted from January to October 2002. As prepubertal children (24 females, 18 males) were defined those aged 9-10 years. Criterion for obesity was body mass index (BMI) greater than 95th percentile of the National Growth Charts, and individuals attending the pediatric and adolescent outpatient clinic of the above-mentioned General Hospital for a basic obesity check-up and fulfilling the above criterion were included in the study as the POC group. BMI was calculated as weight in kilograms divided by height in square meters $\left(\mathrm{kg} / \mathrm{m}^{2}\right)$. Parents were asked to present their children after an overnight fast. None of the children was on any weight management program. At admission, a medical history and physical examination were performed to ensure that the participants were healthy. All prepubertal children were nonsmokers and had normal blood cell counts, serum iron levels, and liver and renal functions as assessed by standard clinical chemistry analyses. No participants were taking multivitamin preparations or medications known to affect lipid metabolism. No dislipidaemia was manifested in relevant electrophoresis. Physical activity of all participants was limited to light gymnastics for 45 minutes, twice a week at school, according to the National School Program. Resting blood pressure was measured in the sitting position after a 15-minute rest using a mercury sphygmomanometer and a cuff appropriately sized for the arm of the subject. Forty-two healthy prepubertal children (21 females, 21 males), on no medications, with BMI less than the 75th percentile on the National Growth Charts, defined as lean (PLC), served as controls. Controls were also nonsmokers and physical activity was also limited to school gymnastics. Controls underwent the same laboratory tests-as examined subjects_-and those tests were normal. Characteristic data of all participants appear on Table 1. Informed consent was obtained from each child and legal guardian.

\section{Analytical Methods}

After an overnight fast, venous blood samples were taken between 7:30 and 9:30 AM. Serum concentrations of CRP were measured by means of high-sensitivity-enhanced immunonephelometry using the Behring Nephelometer System (Behring Diagnostics GmbH, Marburg, Germany). The intra- and interassay coefficients of variation of CRP were $<4.4 \%$ and $<5.7 \%$, respectively, and the analytical sensitivity was $0.18 \mathrm{mg} / \mathrm{L}$. SAA concentrations were measured, in dublicate, by ELISA kit (BioSource International, Camarillo, Calif). The intraand interassay coefficients of variation of SAA were $<7.7 \%$ and $<10.8 \%$, respectively, and the analytical sensitivity was $5 \mathrm{ng} / \mathrm{mL}$. Concentrations of plasma tHcy (homocysteine, disulphide homocysteine, and mixed disulphide homocysteine-cysteine, all in their free and protein bound forms) were assessed in frozen $\left(-70^{\circ} \mathrm{C}\right)$ EDTA plasma on Abbott IMx analyzer using fluorescence polarization immunoassay technology. The intra- and interassay coefficients of variation of tHcy were $1.6 \%$ and $4.9 \%$, respectively, and the analytical sensitivity was $0.16 \mu \mathrm{mol} / \mathrm{L}$ (Abbott Diagnostics, Wiesbaden-Delkenheim, Germany). Cholesterol, triglycerides, and HDL cholesterol were all determined on RA500 automated analyzer (Technicon Instrument Corporation, Tarry Town, NY) according to the manufacturer's recommendations. Serum glucose, creatinine, cholesterol, triglycerides, and HDL cholesterol were all determined on an RA500 automated analyzer (Technicon Instrument Corporation, Tarry Town, NY) according to the manufacturer's recommendations. Serum lowdensity lipoprotein (LDL) cholesterol was estimated using the Friedewald equation. Serum total insulin, vitamin $B_{12}$, and folate were all measured by radioisotopic immunoassays, using the relevant commercial kits (for insulin: Myria Technogenetics, Cassina de Pecchi, Italy, intra- and interassay coefficients of variation: $2.6 \%$ and $4.8 \%$ resp, sensitivity $2.1 \mathrm{pmol} / \mathrm{L}$; for vitamin $\mathrm{B}_{12}$, and folate: DPC, Los Angeles, Calif, intra- and interassay coefficients of variation for vitamin $\mathrm{B}_{12}: 6.6 \%$ and $8.9 \%$ 
TABle 1. Characteristics of the study participants. Data are $n$ or means \pm standard deviation.

\begin{tabular}{|c|c|c|c|}
\hline & Subjects (POC) & Controls (PLC) & $p$ or $P$ \\
\hline$n$ & 72 & 42 & - \\
\hline \multicolumn{4}{|l|}{ Age (years) } \\
\hline Prepubertal children & $8.9 \pm 0.3$ & $9.2 \pm 0.3$ & $p=0.78$ \\
\hline $\operatorname{BMI}\left(\mathrm{kg} / \mathrm{m}^{2}\right)$ & $30.3 \pm 1.1$ & $17.4 \pm 0.5$ & $p=0.0001^{*}$ \\
\hline \multicolumn{4}{|l|}{ Blood pressure $(\mathrm{mmHg})$} \\
\hline Systolic & $124.8 \pm 12.8$ & $122.1 \pm 10.4$ & $p=0.91$ \\
\hline Diastolic & $72.4 \pm 9.9$ & $68.8 \pm 8.6$ & $p=0.48$ \\
\hline Total Homocysteine $(\mu \mathrm{mol} / \mathrm{L})$ & $8.4 \pm 0.5$ & $7 \pm 0.3$ & $p=0.02^{*}$ \\
\hline Triglycerides (mmol/L) & $1.6 \pm 0.8$ & $1.2 \pm 0.8$ & $p=0.02^{*}$ \\
\hline Total cholesterol (mmol/L) & $4.2 \pm 0.9$ & $3.9 \pm 0.9$ & $p=0.63$ \\
\hline HDL cholesterol (mmol/L) & $0.98 \pm 0.27$ & $1.17 \pm 0.3$ & $p=0.003^{*}$ \\
\hline LDL cholesterol (mmol/L) & $2.33 \pm 0.94$ & $2.11 \pm 0.7$ & $p=0.78$ \\
\hline Glucose (mmol/L) & $5.02 \pm 1.41$ & $5.23 \pm 0.9$ & $p=0.65$ \\
\hline Creatinine $(\mu \mathrm{mol} / \mathrm{L})$ & $59.9 \pm 4.7$ & $62.1 \pm 4$ & $p=0.19$ \\
\hline Total insulin $(\mathrm{pmol} / \mathrm{L})$ & $141 \pm 24$ & $102 \pm 19$ & $p=0.05^{*}$ \\
\hline Vit $B_{12}(\mathrm{pmol} / \mathrm{L})$ & $259 \pm 28$ & $331 \pm 35$ & $p=0.09$ \\
\hline Folate $(\mathrm{nmol} / \mathrm{L})$ & $13.2 \pm 5.8$ & $18.9 \pm 8.3$ & $p=0.04^{*}$ \\
\hline
\end{tabular}

* Statistically significant possibility.

resp, sensitivity $0.2 \mathrm{pmol} / \mathrm{L}$, for folate: intra- and interassay coefficients of variation $6.1 \%$ and $7.9 \%$ resp, sensitivity $0.7 \mathrm{nmol} / \mathrm{L})$.

\section{Statistical Analysis}

Variables were checked for normality of their distribution (Shapiro-Wilks test; Lilliefors test if $N \geq 50$ ). Data that were not normally distributed were $\log _{10}$ transformed (total insulin, triglycerides, and glucose). However the arithmetic mean concentrations are reported in the text and tables. An unpaired $t$ test was used to compare the available anthropometric and metabolic characteristics, except tHcy, between POC and PLC. Multiple linear regression was performed three times to evaluate the difference in levels of CRP, SAA, and tHcy, each time, between POC and PLC controlling in all cases for age and sex, as well as $\log _{10}$ total insulin, vit $B_{12}$, folate, total cholesterol, HDL cholesterol, $\log _{10}$ triglycerides, and $\log _{10}$ glucose levels. To evaluate the associations of BMI and SAA levels to CRP levels as well as the association of BMI to SAA levels, multiple linear regression analysis was applied, adjusting for age and sex, as well as $\log _{10}$ total insulin, vit $\mathrm{B}_{12}$, folate, total cholesterol, HDL cholesterol, $\log _{10}$ triglycerides and $\log _{10}$ glucose levels. Multiple linear regression was also applied to investigate the association of CRP and SAA levels to tHcy levels, adjusting for age and sex, as well as $\log _{10}$ total insulin, vit $\mathrm{B}_{12}$, folate, total cholesterol, HDL cholesterol, $\log _{10}$ triglycerides, and $\log _{10}$ glucose levels. The results are expressed as mean \pm standard deviation (SD). All $p$ ( $t$ test) or $P$ (regression analysis) values are two-tailed
TABLE 2. CRP and SAA serum levels of the study participants. Data are expressed as means \pm standard deviation (median).

\begin{tabular}{l|cc}
\hline Parameter & Subjects (POC) & Controls (PLC) \\
\hline CRP $(\mathrm{mg} / \mathrm{L})$ & $3.6 \pm 0.6(3.2)$ & $1.8 \pm 0.7(1.3)$ \\
SAA $(\mathrm{ng} / \mathrm{mL})$ & $12.4 \pm 2.6(11.5)$ & $14.3 \pm 5.7(15.6)$ \\
\hline
\end{tabular}

and $P<.05$ is considered statistically significant. The statistical package SPSS 8.0 (Statistical Package for the Social Sciences, SPSS Inc, Chicago, Ill) was used.

\section{RESULTS}

POC and PLC presented no significant differences for all the rest available anthropometric and common metabolic characteristics, except for BMI, tHcy, triglycerides, and total insulin which were significantly higher in POC than in PLC $(p=0.0001, p=0.02, p=0.02$, and $p=0.05$, resp) and for HDL cholesterol and folate which were significantly lower in POC than in PLC $(p=0.003$ and $p=0.04$, resp) (Table 1). Circulating levels of CRP (A) and SAA (B) for both POC and PLC are shown in Table 2. The mean adjusted (for age and sex, as well as $\log _{10}$ total insulin, vit $B_{12}$, folate, total cholesterol, $\log _{10}$ triglycerides, and $\log _{10}$ glucose levels) differences in CRP and SAA levels between POC and PLC were $3.12 \mathrm{mg} / \mathrm{L}$ (confidence interval (CI): 0.19-6.05 mg/L, $P=0.04$ ) and $3.87 \mathrm{ng} / \mathrm{mL}(\mathrm{CI}:-16.66-24.39 \mathrm{ng} / \mathrm{mL}, P=0.71)$, respectively. 
In the model of analysis used to evaluate the association of BMI and SAA to CRP levels and the association of BMI to SAA levels, OCA presented positive association of age and $\log _{10}$ triglycerides, total cholesterol, glucose, and $\log _{10}$ total insulin levels and negative association of HDL-cholesterol to CRP $(P=.02)$, but not to SAA levels. Thus one $\mathrm{mmol} / \mathrm{L}$ HDL cholesterol decrease was associated to $14.2 \mathrm{mg} / \mathrm{L}(\mathrm{Cl}: 2.9-22.2 \mathrm{mg} / \mathrm{L}$ ) increase in CRP levels. Furthermore, in this group, positive significant association of BMI to CRP levels $(P=.0001)$, but not to SAA levels was noticed. Thus, one $\mathrm{Kg} / \mathrm{m}^{2}$ increase in BMI was associated with $0.44 \mathrm{mg} / \mathrm{L}(\mathrm{Cl}: 0.27-0.61 \mathrm{mg} / \mathrm{L})$ increase in CRP levels. Furthermore, in POC, SAA levels were associated significantly to CRP levels in a positive direction $(P=.02)$. Thus, one $\mathrm{mg} / \mathrm{L}$ increase in CRP levels was associated with a $4.25 \mathrm{ng} / \mathrm{mL}(\mathrm{Cl}: 1.5-5.8 \mathrm{ng} / \mathrm{mL})$ increase in SAA levels.

In the model of analysis applied to investigate the association of BMI, CRP, and SAA levels to tHcy levels, in OCA, BMI as well as CRP and SAA levels were all not associated significantly with tHcy levels.

\section{DISCUSSION}

To our knowledge, this is the first study that extrapolates in POC the profile of the chronic low-grade systemic inflammation with regard to SAA and its possible interrelation to circulating CRP. Furthermore, this study investigates, for the first time in POC, the association among the markers of chronic low-grade systemic inflammation of this specific profile and plasma levels of tHcy.

The data from this study demonstrate that (a) CRP levels are higher in POC than in PLC, (b) SAA levels do not differ between POC and PLC, (c) a positive association of BMI to CRP levels, not to SAA levels, exists in POC, (d) a negative association of HDL cholesterol to CRP levels, not to SAA levels, also exists in POC, and (e) the positively interrelated CRP and SAA levels fail to associate with $\mathrm{tHcy}$ in POC.

The participants of the present study were all under the age of 10 years to avoid any significant interference of puberty-related insulin resistance as well as circulating insulin and glucose levels enhancement, although it has recently been postulated that hyperinsulinemia and insulin resistance are already present in POC [21]. Indeed, in this study POC presented higher insulin levels than those of PLC. Accordingly, adjustment for insulin levels of all the results of the study was performed. However, glucose homeostasis was maintained in POC, since no significant variations in circulating glucose levels were observed between POC and PLC. This probably reflects a potentially reversible hyperinsulinemia indicative of a stage before the onset of puberty-related insulin resistance in POC.

Circulating CRP is not only a widely accepted marker of chronic low-grade systemic inflammation, but moreover it is a direct promoter of vascular disease. A variety of mechanisms by which CRP might promote vascular disease (such as its frequent detection in atherosclerotic plaques, its ability to stimulate tissue factor production by macrophages, and its enhancement of complement activation after binding partly degraded, nonoxidized low density lipoprotein cholesterol) have been proposed, but none is proved. In this study, higher CRP levels were noticed in POC, compared to those noticed in PLC. Moreover, a strong positive association of body mass index (BMI), generally accepted now to be used to define obesity in children and adolescents clinically [1], to CRP levels was noticed only in POC. These results confirm previous relevant findings in children [4] and extend previous research in adults [22] to children. In addition, a negative association of decreased HDL cholesterol levels to CRP was observed. This proatherogenic, noncausative association extends, for the first time, previous results in adults $[23,24]$ to children. Accordingly, excess body weight estimated by BMI is evidently associated with a state of chronic low-grade systemic inflammation in POC.

SAA is a not-often-used marker of chronic low-grade systemic inflammation. However, several findings emphasize the importance of SAA in various physiological and pathological processes, including inflammation and atherosclerosis. Although the precise role of SAA has not been defined, many potential clinically important atherogenic functions have been proposed [15]. These include involvement in lipid metabolism/transport, amyloid A amyloidosis, induction of extracellular-matrix-degrading enzymes, and chemotactic recruitment of inflammatory cells. On the contrary, other data coupled with observations in which SAA was found to induce endothelial prostaglandin $\mathrm{I}_{2}\left(\mathrm{PGI}_{2}\right)$ formation and to inhibit overproduction of $\mathrm{PGI}_{2}$ by TNF and lipopolysacharides (LPS) as well as platelet aggregation may suggest that SAA contributes to the protective effect of HDL cholestrol against atherosclerosis.

Several studies reported elevation of SAA levels in unstable coronary syndromes $[11,15]$ and spontaneous or transplant-associated coronary artery disease [11]. Accompanied by circulating CRP elevation, circulating SAA elevation has been reported in type-2 diabetic patients [25], in patients with coronary heart disease [26], and in patients at risk for future coronary occlusion [13]. However, some studies have suggested that SAA levels are not necessarily accompanied by CRP levels in any changes. Accordingly, circulating SAA but not CRP elevation was observed in patients with mild to moderate essential hypertension [14]. In addition, the extent and severity of atherosclerosis of left coronary arteries, evaluated by Gensini Score, correlated with the percentage changes in SAA across coronary circulation but not with changes in CRP [5]. On the other hand, SAA levels are not associated with peripheral artery disease, in contrary to CRP levels which are associated with lower summary performance score among patients with peripheral artery disease [27]. In this study, SAA levels did not differ between 
POC and PLC. Combined with the increased CRP levels, this result may indicate that control mechanisms separately permit circulating CRP elevation but not SAA elevation in POC. This is likely to be of relevance to the causation of future atherosclerosis.

An important observation of the present study is that decreased HDL cholesterol are not associated to unaltered SAA levels in POC. It has been previously reported [28] that raised SAA lowers HDL cholesterol levels causatively, through association with lipoproteins of the high-density range, which alters HDL cholestrol efflux, to be the principal role of SAA. Thus, the unrelated to decreased HDL cholesterol levels, unaltered SAA levels may limit the proatherogenic potential of the related to decreased HDL cholestrol, raised CRP levels. In addition, no association of BMI to SAA levels was noticed in POC. The lack of this association is a differential finding for POC, since association of BMI to SAA levels has been described in type-2 diabetic patients [25] and even more in healthy adults without history of coronary artery disease [29] as well as in healthy women [22]. As circulating levels of both CRP and SAA have been separately associated with increased risk of coronary heart disease, the close interrelation between CRP and SAA levels observed in POC increases the likelihood that there is some underlying process related to inflammation which is relevant to the causation of the disease [13].

These results revealed, for the first time, a differential, in comparison to other subpopulations studied $[5,13,14$, 15], profile of chronic low-grade systemic inflammation in that, although close interrelated, only CRP levels, negatively related to decreased HDL cholesterol, not SAA levels, unrelated to decreased HDL cholesterol, are elevated in POC.

Confirming several previous findings in both adults and children $[19,21]$, we recently demonstrated $[20]$ an unrelated to BMI, mild elevation of tHcy levels in POC as compared to tHcy levels measured in PLC, which might be regarded as an indicator of the global cardiovascular risk. The present study failed to observe an association of CRP or SAA levels to tHcy levels in POC. This finding extends for the first time previous finding in adults to children and may imply that prepubertal childhood obesity-specific chronic low-grade systemic inflammation does not contribute to homocysteinemia-induced promotion of atherosclerosis.

A shortcoming of this study might be that vitamin $B_{6}$, insulin resistance, total antioxidant status, as well as leptin levels were not measured. Some of them such as vita$\min \mathrm{B}_{6}$ and insulin resistance have been reported as determinants for circulating markers of chronic low-grade inflammation, including CRP, but they all represent only some of the determinants for plasma levels of tHcy [30]. In most cases, an abnormal CRP or tHcy status is not caused by a single factor alone but often is the result of combined effects. However, it was not only out of the pur- pose of this study, but also it was impossible to evaluate all the known determinants of CRP and SAA or tHcy levels in the limits of a single study. Moreover, the subjects of this study, except for being obese, were all biochemically and clinically, apparently healthy.

In conclusion, this study provides evidence that in prepubertal childhood obesity a unique profile of chronic low-grade systemic inflammation in that, although they are closely interrelated, only CRP, negatively related to decreased HDL cholesterol, not SAA levels, unrelated to decreased HDL cholesterol, are elevated in POC, is evoked. This POC-specific chronic low-grade systemic inflammation cannot explain the link between homocysteinemia, observed in childhood obesity, and future atherosclerosis.

\section{REFERENCES}

[1] Kiess W, Galler A, Reich A, et al. Clinical aspects of obesity in childhood and adolescence. Obes Rev. 2001;2(1):29-36.

[2] Desideri G, De Simone M, Iughetti L, et al. Early activation of vascular endothelial cells and platelets in obese children. J Clin Endocrinol Metab. 2005;90(6):3145-3152.

[3] Das UN. Is obesity an inflammatory condition? $\mathrm{Nu}$ trition. 2001;17(11-12):953-966.

[4] Ford ES, Galuska DA, Gillespie C, Will JC, Giles WH, Dietz WH. C-reactive protein and body mass index in children: findings from the Third National Health and Nutrition Examination Survey, 1988-1994. J Pediatr. 2001;138(4):486-492.

[5] Matsubara T, Ishibashi T, Hori T, et al. Association between coronary endothelial dysfunction and local inflammation of atherosclerotic coronary arteries. Mol Cell Biochem. 2003;249(1-2):67-73.

[6] Svatikova A, Wolk R, Shamsuzzaman AS, Kara T, Olson EJ, Somers VK. Serum amyloid A in obstructive sleep apnea. Circulation. 2003;108(12):14511454.

[7] Malle E, De Beer FC. Human serum amyloid A (SAA) protein: a prominent acute-phase reactant for clinical practice. Eur J Clin Invest. 1996;26(6):427435.

[8] Wilkins J, Gallimore JR, Moore EG, Pepys MB. Rapid automated high sensitivity enzyme immunoassay of C-reactive protein. Clin Chem. 1998;44(6 pt1): 1358-1361.

[9] Wilkins J, Gallimore JR, Tennent GA, et al. Rapid automated enzyme immunoassay of serum amyloid A. Clin Chem. 1994;40(7 pt 1):1284-1290.

[10] Valle M, Martos R, Gascon F, Canete R, Zafra MA, Morales R. Low-grade systemic inflammation, hypoadiponectinemia and a high concentration of leptin are present in very young obese children, and correlate with metabolic syndrome. Diabetes Metab. 2005;31(1):55-62. 
[11] Fyfe AI, Rothenberg LS, DeBeer FC, Cantor RM, Rotter JI, Lusis AJ. Association between serum amyloid A proteins and coronary artery disease: evidence from two distinct arteriosclerotic processes. Circulation. 1997;96(9):2914-2919.

[12] Retterstol L, Eikvar L, Berg K. A twin study of C-reactive protein compared to other risk factors for coronary heart disease. Atherosclerosis. 2003;169 (2):279-282.

[13] Danesh J, Whincup P, Walker M, et al. Low grade inflammation and coronary heart disease: prospective study and updated meta-analyses. BMJ. 2000;321 (7255):199-204.

[14] Economou E, Pitsavos C, Stefanadis C, et al. Serum amyloid A levels in patients with mild to moderate hypertension, before and after treatment with a calcium antagonist. Im: American College of Cardiology 48th Annual Scientific Session; March, 1999; New Orleans, La. Abstract 1223-1272.

[15] Kisilevsky R, Tam SP. Acute phase serum amyloid A, cholesterol metabolism, and cardiovascular disease. Pediatr Pathol Mol Med. 2002;21(3):291-305.

[16] Hackam DG, Anand SS. Emerging risk factors for atherosclerotic vascular disease: a critical review of the evidence. JAMA. 2003;290(7):932-40.

[17] Stehouwer CD, Weijenberg MP, van den Berg M, Jakobs C, Feskens EJ, Kromhout D. Serum homocysteine and risk of coronary heart disease and cerebrovascular disease in elderly men: a 10-year followup. Arterioscler Thromb Vasc Biol. 1998;18(12):18951901.

[18] Gallistl S, Sudi KM, Erwa W, Aigner R, Borkenstein M. Determinants of homocysteine during weight reduction in obese children and adolescents. Metabolism. 2001;50(10):1220-1223.

[19] Gallistl S, Sudi K, Mangge H, Erwa W, Borkenstein $\mathrm{M}$. Insulin is an independent correlate of plasma homocysteine levels in obese children and adolescents. Diabetes Care. 2000;23(19):1348-1352.

[20] Economou EV, Malamitsi-Puchner AV, Pitsavos CP, et al. Negative association between circulating total homocysteine and proinflammatory chemokines MCP-1 and RANTES in prepubertal lean, but not in obese, children. J Cardiovasc Pharmacol. 2004;44(3): 310-315.

[21] Galli-Tsinopoulou A, Karamouzis M, NousiaArvanitakis S. Insulin resistance and hyperinsulinemia in prepubertal obese children. J Pediatr Endocrinol Metab. 2003;16(4):555-560.

[22] Heilbronn LK, Noakes M, Clifton PM. Energy restriction and weight loss on very-low-fat diets reduce C-reactive protein concentrations in obese, healthy women. Arterioscler Thromb Vasc Biol. 2001;21(6): 968-970.
[23] Kazumi T, Kawaguchi A, Hirano T, Yoshino G. Creactive protein in young, apparently healthy men: associations with serum leptin, QTc interval, and high-density lipoprotein-cholesterol. Metabolism. 2003;52(9):1113-1116.

[24] Pirro M, Siepi D, Lupattelli G, et al. Plasma Creactive protein in subjects with hypo/hyperalphalipoproteinemias. Metabolism. 2003;52(4):432436.

[25] Leinonen E, Hurt-Camejo E, Wiklund O, Hulten LM, Hiukka A, Taskinen MR. Insulin resistance and adiposity correlate with acute-phase reaction and soluble cell adhesion molecules in type 2 diabetes. Atherosclerosis. 2003;166(2):387-394.

[26] Jousilahti P, Salomaa V, Rasi V, Vahtera E, Palosuo T. The association of c-reactive protein, serum amyloid a and fibrinogen with prevalent coronary heart disease-baseline findings of the PAIS project. Atherosclerosis. 2001;156(2):451-456.

[27] McDermott MM, Greenland P, Green D, et al. Ddimer, inflammatory markers, and lower extremity functioning in patients with and without peripheral arterial disease. Circulation. 2003;107(25):31913198.

[28] Yudkin JS, Kumari M, Humphries SE, MohamedAli V. Inflammation, obesity, stress and coronary heart disease: is interleukin-6 the link? Atherosclerosis. 2000;148(2):209-214.

[29] Danesh J, Muir J, Wong YK, Ward M, Gallimore JR, Pepys MB. Risk factors for coronary heart disease and acute-phase proteins. A population-based study. Eur Heart J. 1999;20(13):954-959.

[30] Gomez-Ambrosi J, Salvador J, Paramo JA, et al. Involvement of leptin in the association between percentage of body fat and cardiovascular risk factors. Clin Biochem. 2002;35(4):315-320. 


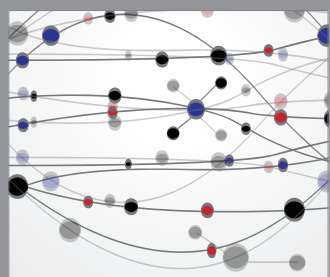

The Scientific World Journal
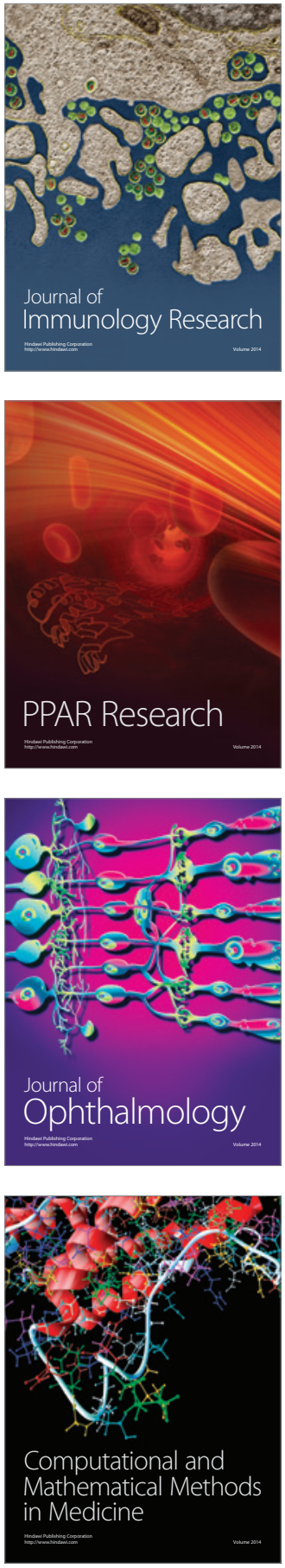

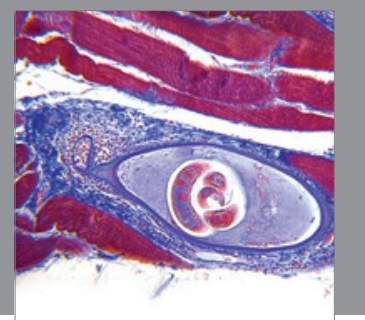

Gastroenterology

Research and Practice
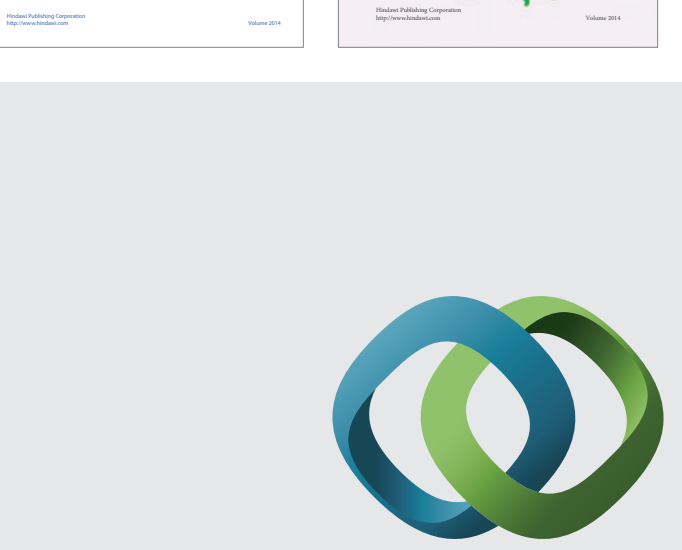

\section{Hindawi}

Submit your manuscripts at

http://www.hindawi.com
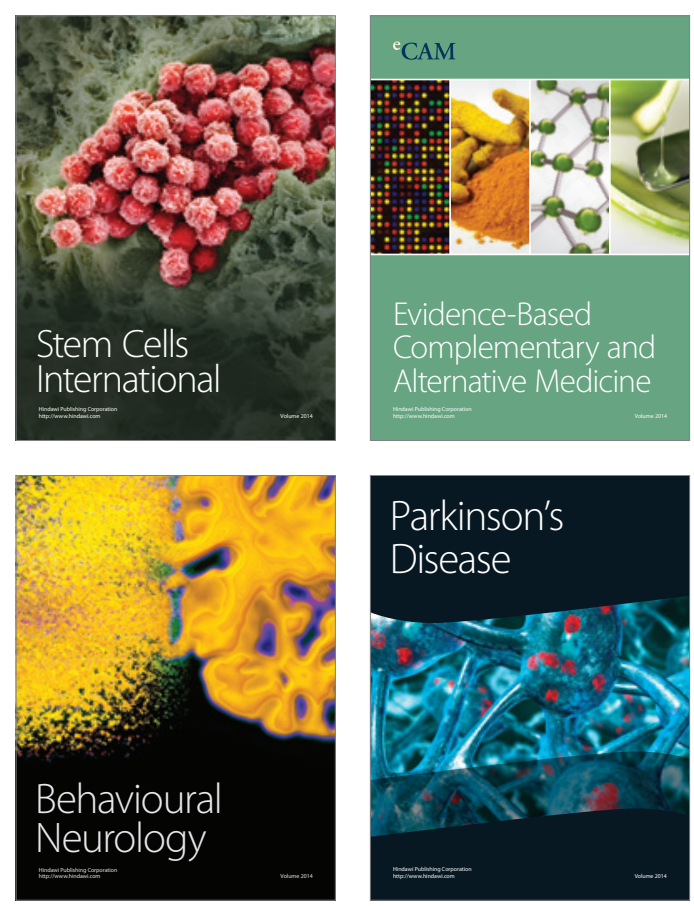

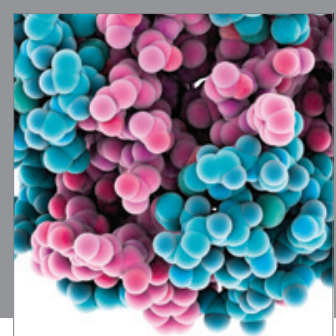

Journal of
Diabetes Research

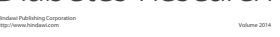

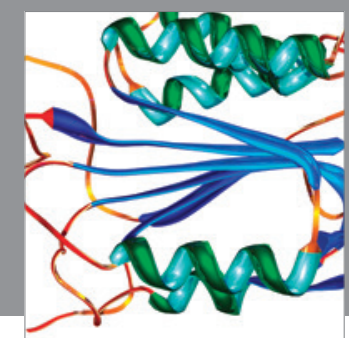

Disease Markers
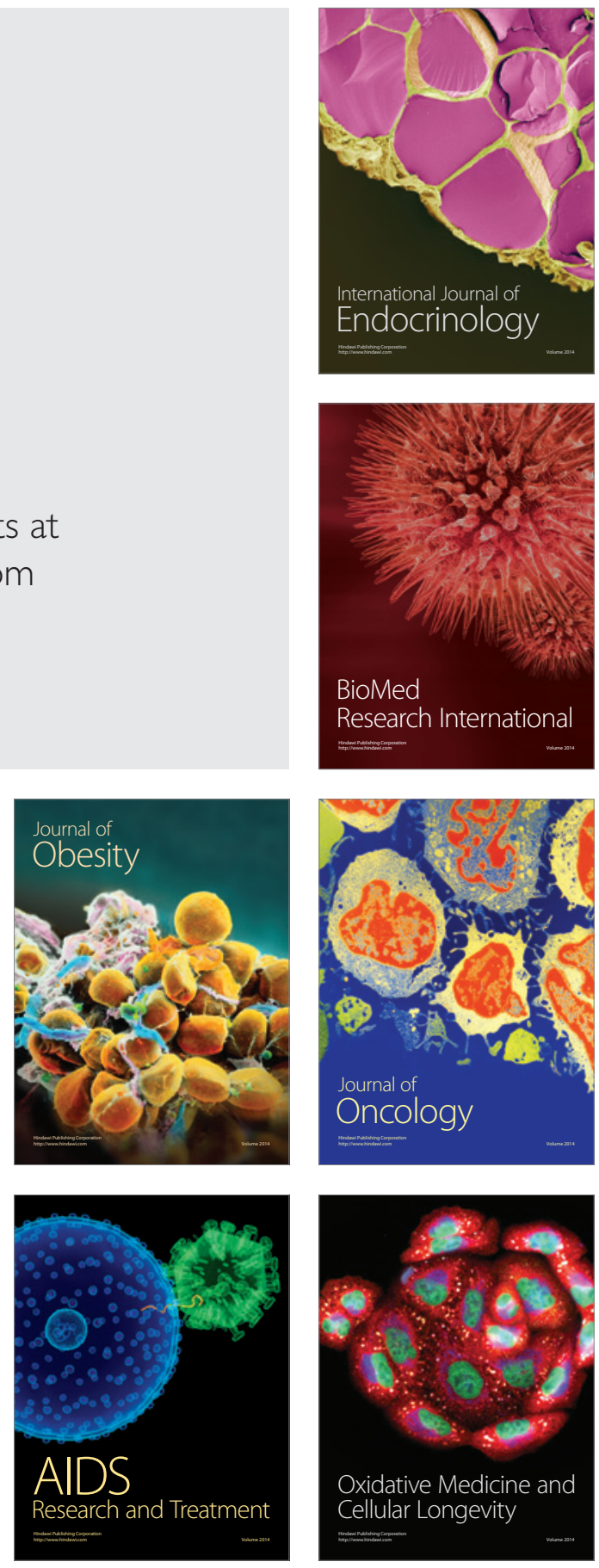